\title{
Particularities of the directed flow in the NICA energy range
}

\author{
Viacheslav Toneev* \\ Joint Institute for Nuclear Research, Dubna \\ E-mail: toneevatheor.jinr.ru
}

\section{Volodymyr Konchakovski}

Institute for Theoretical Physics, University of Giessen, Giessen

E-mail: Konchakovski@theo.physik.uni-giessen.de

\section{Wolfgang Cassing}

Institute for Theoretical Physics, University of Giessen, Giessen

E-mail: Wolfgang.Cassing@theo.physik.uni-giessen.de

\section{Yuri B. Ivanov}

Kurchatov Institute \& National Research Nuclear University "MEPhI", Moscow

E-mail: Y.Ivanov@gsi.de

\begin{abstract}
Recent STAR data for the directed flow of protons, antiprotons and charged pions obtained within the beam energy scan program are analyzed within the Parton-Hadron-String-Dynamics (PHSD/HSD) transport models and a 3-Fluid hydroDynamics (3FD) approach. Both versions of the kinetic approach, HSD and PHSD, are used to clarify the role of partonic degrees of freedom. The PHSD results, simulating a partonic phase and its coexistence with a hadronic one, are roughly consistent with data. The hydrodynamic results are obtained for two Equation of States (EoS), a pure hadronic EoS and an EoS with a crossover type transition. The latter case is favored by the STAR experimental data. Special attention is paid to the description of antiproton directed flow based on the balance of $p \bar{p}$ annihilation and the inverse processes for $p \bar{p}$ pair creation from multi-meson interactions. Generally, the semi-qualitative agreement between the measured data and model results supports the idea of a crossover type of quark-hadron transition which softens the nuclear EoS but shows no indication of a first-order phase transition.
\end{abstract}

XXII International Baldin Seminar on High Energy Physics Problems 15-20 September, 2014

JINR, Dubna, Russia

\footnotetext{
${ }^{*}$ Speaker.
} 


\section{Introduction}

As has been widely recognized, the study of the particle azimuthal distribution in momentum space with respect to the reaction plane is an important tool to probe the hot, dense matter created in heavy-ion collisions $[1,2]$. The directed flow refers to a collective sidewards deflection of particles and is characterized by the first-order harmonic $v_{1}$ of the Fourier expansion of the particle azimuthal angular distribution with respect to the reaction plane [3]. The second harmonic coefficient $v_{2}$, called elliptic flow, and the triangular flow $v_{3}$ have been extensively studied both theoretically and experimentally in the last years by about five orders of magnitude in the collision energy $\sqrt{s_{N N}}$ [4]. In contrast, apart from first measurements in the early nineties and till recent times, the directed flow was studied mainly theoretically although some experimental information from the SchwerionenSynchrotron (SIS) to Super-Proton-Synchrotron (SPS) energies is available [5].

It is generally believed that the directed transverse flow is generated early in the heavy-ion collision before a thermalization of the degrees of freedom occurs. In particular, in the fragmentation region (i.e. at large rapidity or pseudorapidity), the directed flow is generated during the nuclear passage time $[6,7]$. The directed transverse flow therefore probes the onset of bulk collective dynamics during thermalization, thus providing valuable information on the pre-equilibrium stage $[8,9,10,11]$. In earlier times (at moderate beam energies) the first flow harmonic defined as

$$
v_{1}(y)=\left\langle\cos \left(\phi-\phi_{R P}\right)\right\rangle=\left\langle v_{x} / \sqrt{v_{x}^{2}+v_{y}^{2}}\right\rangle
$$

with respect to the reaction plane $\phi_{R P}$ was characterized differently: i.e. by the mean transverse momentum per particle projected on the reaction $(x-z)$ plane $\left\langle p_{x}(y) / N\right\rangle$ in the center-of-mass system which differs from the $v_{1}$ harmonic component. Unfortunately, it is not possible to convert or directly compare $v_{1}$ data to the earlier $p_{x} / N$ analysis. Often, just the slope of $v_{1}(y)$ at midrapidity has been used to quantify the strength of the directed flow. The shape of the rapidity dependence $v_{1}(y)$ is of special interest because the directed flow at midrapidity may be modified by the collective expansion and reveal a signature of a phase transition from normal nuclear matter to a quark-gluon plasma (QGP) and reflects important features of the system evolution from its initial state. Situation in this field is shortly reviewed in [12].

It is an experimental challenge to measure accurately $v_{1}(y)$ at RHIC energies due to the relatively small signal and a potentially large systematic error arising from azimuthal correlations not related to the reaction plane orientation (non-flow effects). The first RHIC measurements of azimuthal anisotropy for charged particles at $\sqrt{s_{N N}}=(62-200) \mathrm{GeV}$ show that $v_{1}(y)$ appears to be close to zero near midrapidity. Similar results have been obtained by the STAR [13], PHOBOS [14] and PHENIX collaborations using different correlation methods. The model analysis of these data for non-identified hadrons is in a reasonable agreement with experiment and shows no wiggle structure $[15,16]$. Generally, similar conclusions follow from the analysis of the $v_{1}(y)$ excitation functions in a large energy range carried out within different macroscopic (hydro with hadronic, two-phase and chiral transition EoS $[15,17,18]$ ) and microscopic (UrQMD and multiphase transport $[15,19,20]$ ) models which definitely show that systematic measurements with higher precision for identified hadrons and more developed models are needed.

The interest in the directed flow $v_{1}(y)$ has recently been enhanced considerably due to new STAR data obtained in the framework of the beam energy scan (BES) program [21]. The directed 
flow of identified hadrons - protons, antiprotons, positive and negative pions - has been measured with high precision for semi-central Au+Au collisions in the energy range $\sqrt{s_{N N}}=(7.7-39) \mathrm{GeV}$. These data provide a promising basis for studying direct-flow issues as discussed above and have been addressed already by the Frankfurt group [22] limiting themselves to the energy $\sqrt{s_{N N}}<20$ $\mathrm{GeV}$ where hadronic processes are expected to be dominant. However, the authors of Ref. [22] did not succeed to describe the data and to obtain conclusive results which led to the notion of the 'directed flow puzzle'. Our study aims to analyze these STAR results in the whole available energy range including in particular antiproton data. Here we use two complementary approaches: the kinetic transport (the parton-hadron string dynamics (PHSD)) approach and relativistic three-fluid hydrodynamics (3FD) with different equations of state.

We start with a short presentation of the PHSD approach and its hadronic version HSD (without partonic degrees of freedom) and then analyse the BES data in terms of both transport models in order to explore where effects from partonic degrees of freedom show up. Furthermore, we compare also with predictions of other kinetic models in Sec. II while in Sec. III a similar analysis is performed within a collective model, i.e. the 3 fluid hydrodynamics. Our findings are summarized in Sec. IV.

\section{Directed flow in microscopic approaches}

\subsection{Reminder of PHSD}

The PHSD model is a covariant dynamical approach for strongly interacting systems formulated on the basis of Kadanoff-Baym equations [23, 24] or off-shell transport equations in phasespace representation, respectively. In the Kadanoff-Baym theory the field quanta are described in terms of dressed propagators with complex selfenergies. The PHSD model includes the creation of massive quarks via hadronic string decay - above the critical energy density $\sim 0.5 \mathrm{GeV} / \mathrm{fm}^{3}$ - and quark fusion forming a hadron in the hadronization process. With some caution, the latter process can be considered as a simulation of a crossover transition since the underlying EoS in PHSD is a crossover [25]. For a review on off-shell transport theory we refer the reader to Ref. [25].

Fig. 1 illustrates how the hadron multiplicity $d N / d y(y=0)$ at midrapidity is reproduced within the PHSD (solid lines) and HSD (dashed lines) kinetic approaches. We point out that the antiproton abundance is a crucial issue. In the AGS-SPS low energy range $(\approx 20 \mathrm{GeV})$ both models agree quite reasonably with experiment, including the antiproton yield. The enhancement of the proton and antiproton yield at $\sqrt{s_{N N}}=62 \mathrm{GeV}$ in PHSD relative to HSD can be traced back to a larger baryon/antibaryon fraction in the hadronization process. At lower energies this agreement is reached by taking into account the $p \bar{p}$ annihilation to three mesons (e.g. $\pi, \rho, \omega)$ as well as the inverse channels employing detailed balance as worked out in Ref. [30]. These inverse channels are quite important; in particular, at the top SPS energy this inverse reaction practically compensates the loss of antiprotons due to their annihilation [30]. At lower SPS and AGS energies the annihilation is dominant due to the lower meson abundancies, however, the backward channels reduce the net annihilation rate. We mention that the multiple-meson recombination channels are not incorporated in the standard UrQMD transport model [31]. The proton multiplicities are reproduced rather well in the PHSD/HSD approaches but the multiplicity of charged pions is slightly overestimated 


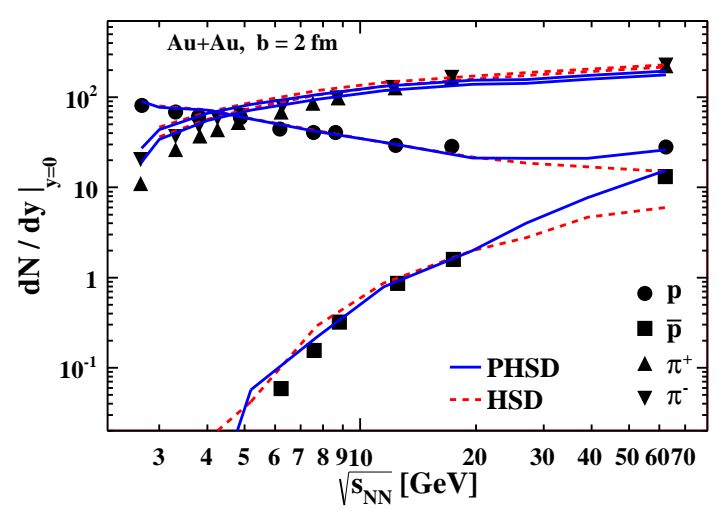

Figure 1: Particle abundance at mid-rapidity calculated for central collisions $b=2 \mathrm{fm}$ in the HSD (dashed lines) and PHSD (solid lines) models. The experimental data are from a compilation of Ref. [27] complemented by recent data from the STAR collaboration [28] and the latest update of the compilation of NA49 results [29].

for $\sqrt{s_{N N}} \lesssim 10 \mathrm{GeV}$. This discrepancy is observed also in other transport modelsand is a subject of separate investigations.

\subsection{Directed flow from microscopic dynamical models}

The whole set of directed flow excitation functions for protons, antiprotons and charged pions from the PHSD/HSD models is presented in Fig. 2 in comparison to the measured data [21]. The initial states in the PHSD/HSD are simulated on an event-by-event basis taking into account fluctuations in the position of the initially colliding nucleons and fluctuations in the reaction plane. This procedure is identical to that in the study of the elliptic flow in Ref. [26]. The average impact parameter for the selected events is $b=7 \mathrm{fm}$. In the simulations the experimental acceptance $0.2 \geq p_{T} \geq 2 \mathrm{GeV} / \mathrm{c}$ is taken into account for all hadrons [21].

At first glance, both models - in particularly the PHSD - correctly reproduce the general trends in the differential $v_{1}(y)$ with bombarding energy: the $v_{1}(y)$ slope for protons is positive at low energies $\left(\sqrt{s_{N N}} \leq 20 \mathrm{GeV}\right)$ and approaches zero with increasing energy while antiprotons and pions have negative slopes, respectively, in the whole energy range. In more detail: for protons the directed flow distributions are in a reasonable agreement with the STAR measurements in the whole range of the collision energies considered (except for $\sqrt{s_{N N}}=11.5 \mathrm{GeV}$ ). However, $v_{1}(y)$ for antiprotons agrees with the data only for the highest energies where baryon/antibaryon pairs are dominantly produced by hadronization.

The shape of the $v_{1}(y)$ distribution for antiprotons starts progressively to differ from the measured data if we proceed from $\sqrt{s_{N N}}=11.5$ to $7.7 \mathrm{GeV}$. In the lower energy range the HSD and PHSD results get very close which indicates the dominance of hadronic reaction channels (absorption and recreation). The direct flow distributions for negative and positive pions are close to each other and also begin to disagree with experiment in the same range of low collision energies as for antiprotons (see Fig. 2). Again the PHSD results are very close to the experimental measurements at higher energies while the HSD results deviate more sizeably thus stressing the role of partonic degrees of freedom in the entire collision dynamics. The clear overestimation of the $\bar{p}$ and $\pi^{-}$ 


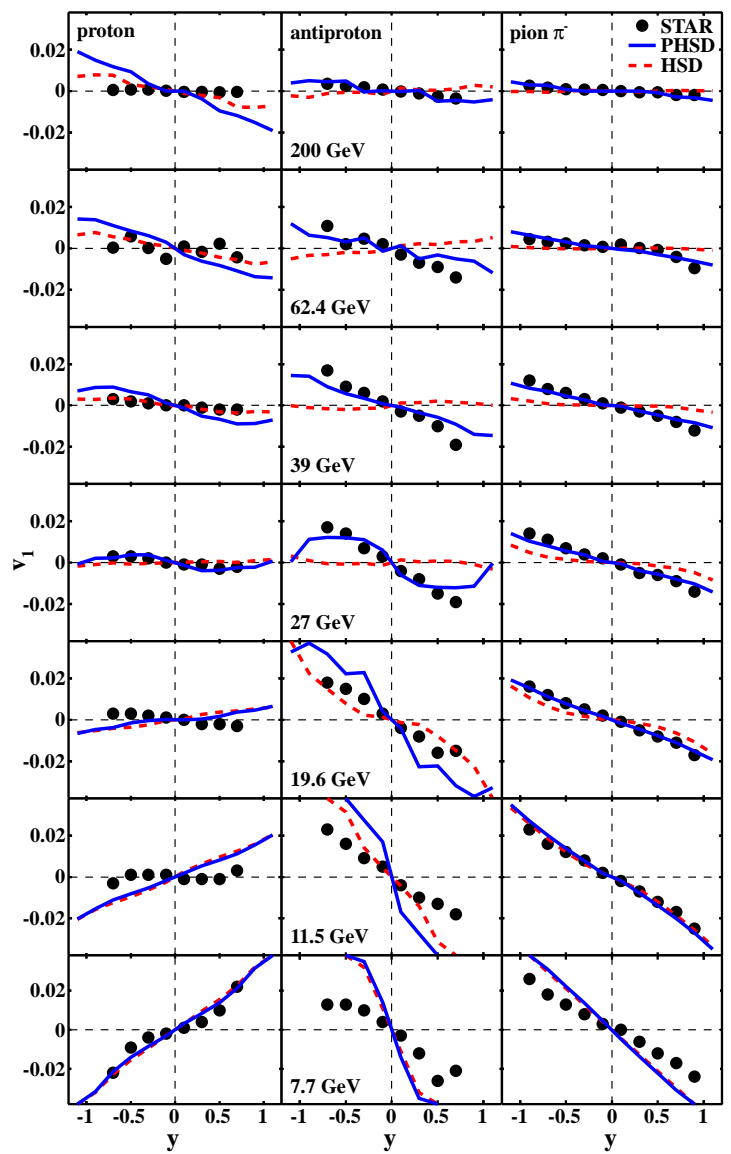

Figure 2: The directed flow $v_{1}(y)$ for protons, antipproton as well as negative pions from Au+Au collisions at different collision energies from $\sqrt{s_{N N}}=7.7$ to $39 \mathrm{GeV}$ from HSD (dashed lines) and PHSD (solid lines). Experimental data are from the STAR collaboration [21].

slopes at $\sqrt{s_{N N}}=7.7 \mathrm{GeV}$ demonstrates that the heavy-ion dynamics is not yet fully understood within the string/hadron picture at the lower energies.

The characteristic slope of the $v_{1}(y)$ distributions at midrapidity, $\left.\frac{d v_{1}}{d y}\right|_{y=0}=F$, is presented in Fig. 3 for all cases considered in Fig. 2. In a first approximation the $v_{1}$ flow in the centerof-mass system may be well fitted by a linear function $v_{1}(y)=F y$ within the rapidity interval $-0.5<y<0.5$. A cubic equation is also used,

$$
v_{1}(y)=F y+C y^{3}
$$

to obtain an estimate of the uncertainty in extracting the coefficient $F$. The error bars in Fig. 3 just stem from the different fitting procedures. Note that the energy axis in Fig. 3 is extended by adding experimental results for $\sqrt{s_{N N}}=62$ and $200 \mathrm{GeV}$ [21]. This representation is more delicate as compared to $v_{1}(y)$ in Fig. 2. For protons there is a qualitative agreement of the HSD/PHSD results with the experiment measurements: the slope $F>0$ at low energies, however, exceeding the experimental values by up a factor of about two; the slope crosses the line $F=0$ at $\sqrt{s_{N N}} \sim 20$ $\mathrm{GeV}$, which is twice larger than the experimental crossing point, and then stays negative and almost constant with further energy increase. However, the absolute values of the calculated proton slopes 


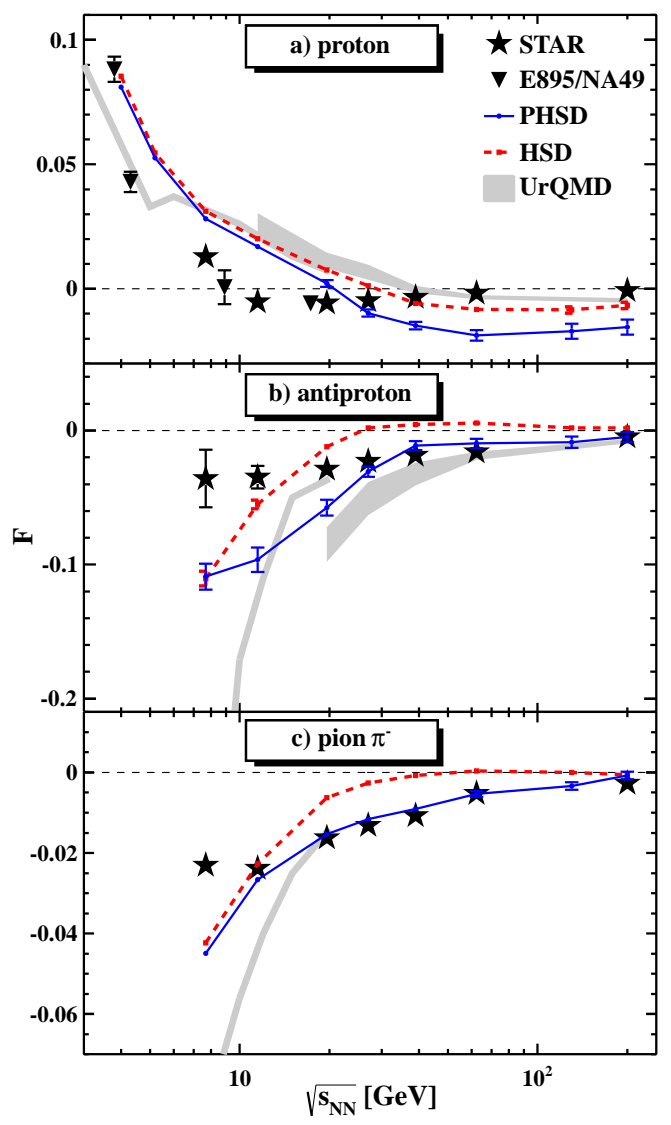

Figure 3: The beam energy dependence of the directed flow slope at midrapidity for protons, antiproton and charged pions from semicentral Au+Au collisions. The shaded band corresponds to the UrQMD results as cited in [21]. The experimental data are from the STAR collaboration [21] along with results of prior experiments using comparable cuts $[32,33]$.

in this high energy range are on the level of -(0.010-0.015), while the measured ones are about -0.005. The standard UrQMD model results, as cited in the experimental paper [21] and in the more recent theoretical work [22], are displayed in Fig. 3 by the wide and narrow shaded areas, respectively. These results for protons are close to those from the HSD and essentially overestimate the slope for energies below $\sim 30 \mathrm{GeV}$ but at higher energy become negative and relatively close to the experiment. The predictions for the pure hadronic version of the transport model HSD (dotted lines in Fig. 3(a)) slightly differ from the PHSD results which overpredict the negative proton slope at higher RHIC energies.

For the antiproton slopes we again observe an almost quantitative agreement with the BES experiment [21]: with increasing collision energy the HSD and PHSD slopes grow and then flatten above $20-30 \mathrm{GeV}$. The HSD results saturate at $v_{1}(0)=0$, while the PHSD predictions stay negative and in good agreement with experiment (see Fig. 3(b)). It is noteworthy to point out that these PHSD predictions strongly differ from the UrQMD results which no longer describe the data for $\sqrt{s_{N N}} \leqslant 20 \mathrm{GeV}$ but are in agreement with the measurements for higher energies. This disagreement might be attributed to a neglect of the inverse processes for antiproton annihilation [30] in UrQMD as described above. 

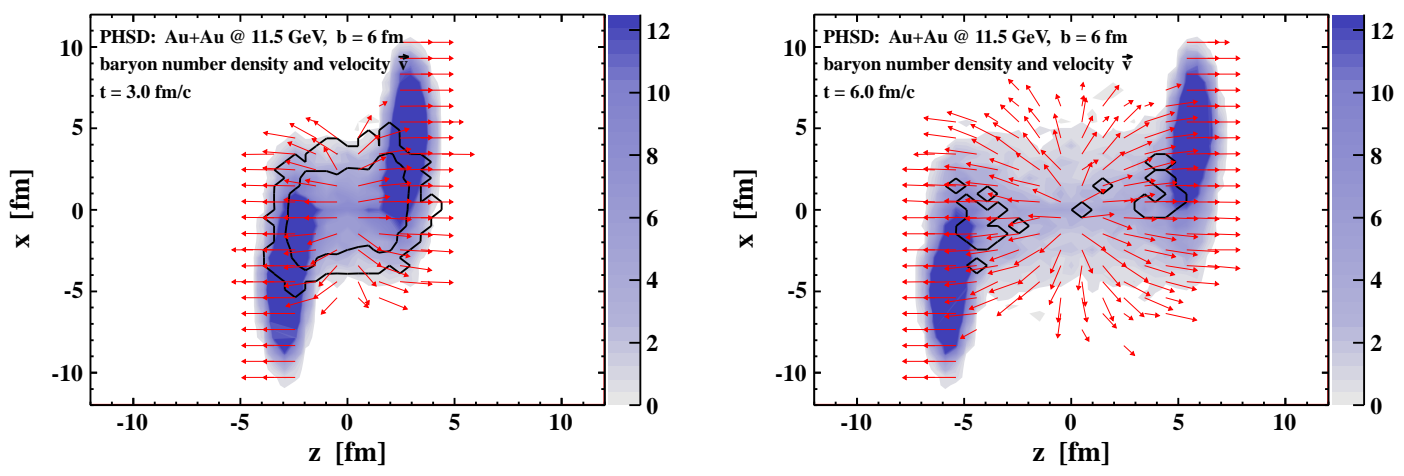

Figure 4: Snapshots of the baryon energy density distribution in the PHSD model at the time $t=3 \mathrm{fm} / \mathrm{c}$ and $6 \mathrm{fm} / \mathrm{c}$ for $\mathrm{Au}+\mathrm{Au}$ collisions and $\sqrt{s_{N N}}=11.5 \mathrm{GeV}$. The energy density scale is given on the right side in $\mathrm{GeV} / \mathrm{fm}^{3}$. The solid curves display parton density levels for 0.6 and 0.01 partons $/ \mathrm{fm}^{3}$. The arrows show the local velocity of baryonic matter (in relative units).

The differences between the calculations and experimental data become apparent for the charged pion slopes at $\sqrt{s_{N N}} \lesssim 11 \mathrm{GeV}$ : the negative minimum of the charged pion slope is deeper than the measured one. The HSD and PHSD results practically coincide at low energy (due to a minor impact of partonic degrees of freedom) but dramatically differ from those of the UrQMD model for $\sqrt{s_{N N}} \lesssim 20 \mathrm{GeV}$ (see Fig.3(c)). This difference might be attributed again to a neglect of the inverse processes for antiproton annihilation in UrQMD.

The appearance of negative $v_{1}$-slopes can be explained by the evolution of the tilted ellipsoidlike shape of the participant zone as assumed in Refs. [34, 35]. This situation is illustrated in Fig. 4 by PHSD calculations. Snapshots of the velocity profile are shown for times $t=3 \mathrm{fm} / \mathrm{c}$ and $6 \mathrm{fm} / \mathrm{c}$ for semi-peripheral $\mathrm{Au}+\mathrm{Au}(11.5 \mathrm{GeV})$ collisions in the background of baryon density distributions where also parton blobs can be identified. Indeed, among the scattered particles there are many which move perpendicularly to the stretched matter (antiflow) and their multiplicity increases with time. However, this component is weak and it is not clear whether these snapshots will result in observable effects for the final slope.

Thus, in agreement with the STAR experimental data, in the considered energy range the PHSD model predicts for protons a smooth $F\left(\sqrt{s_{N N}}\right)$ function which is flattening at $\sqrt{s_{N N}} \gtrsim 10$ $\mathrm{GeV}$ and reveals no signatures of a possible first order phase transition as expected in Refs. [36, 37, 38]. For antiprotons the slope at midrapidity manifests a wide but shallow negative minimum for $\sqrt{s_{N N}} \approx 30 \mathrm{GeV}$ while the measured slope is a monotonically increasing function. It is noteworthy that the new STAR data are consistent with the PHSD results which include a crossover transition by default due to a matching of the EoS to lattice QCD results.

\section{Directed flow in a macroscopic approach}

\subsection{The 3FD model}

The 3FD model [39] is a straightforward extension of the 2-fluid model with a radiation of direct pionsand (2+1)-fluid model as noted in [25]. These models have been extended to treat the 
baryon-free fluid on an equal footing with the baryon-rich ones. A certain formation time, $\tau$, is allowed for the fireball fluid, during which the matter of the fluid propagates without interactions. The formation time $\tau$ is associated with the finite time of string formation and decay and is incorporated also in the kinetic transport models such as PHSD/HSD.

Contrary to the conventional hydrodynamics, where a local instantaneous stopping of projectile and target matter is assumed, the specific feature of the 3FD is a finite stopping power resulting in a counter-streaming regime of leading baryon-rich matter. The basic idea of a 3FD approximation to heavy-ion collisions $[40,41]$ is that at each space-time point a generally nonequilibrium distribution of baryon-rich matter can be represented as a sum of two distinct contributions initially associated with constituent nucleons of the projectile and target nuclei. Therefore, the 3FD approximation is a minimal way to simulate the finite stopping power at high incident energies.

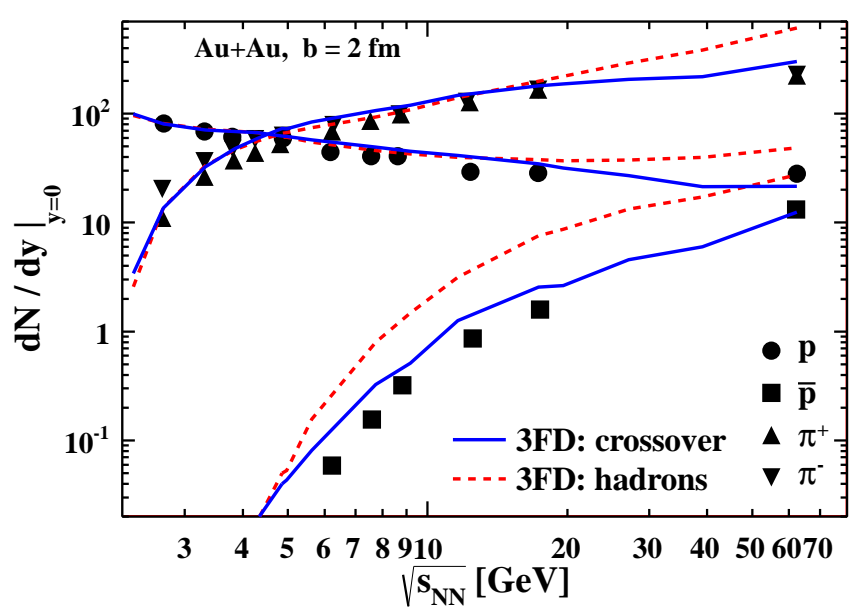

Figure 5: Particle abundance at midrapidity calculated for central collisions $(b=2 \mathrm{fm})$ in the 3FD model with an EoS for a pure hadronic phase (dashed lines) and for the case of a crossover transition (solid lines). The experimental data are the same as in Fig. 1.

Different EoS's can be implemented in the 3FD model in contrast to the PHSD that incorporates only a crossover transition. In particular, in this work we apply a purely hadronic EoS [42] and an EoS with a crossover transition as constructed in Ref. [43]. The physical input of the 3FD calculations is described in detail in Ref. [44]. No tuning (or change) of 3FD-model parameters has been done in the present study as compared to that stated in Ref. [44].

The particle yield at midrapidity calculated within the 3FD model is presented in Fig. 5. Both the hadronic-EoS (dashed lines) and crossover-EoS results (solid lines) for the proton and pion abundancies at $\sqrt{s_{N N}} \leqslant 20 \mathrm{GeV}$ are in good agreement with the experimental data and, in the case of charged pions, in even better agreement than in the HSD/PHSD approaches (cf. Fig. 1). The purely hadronic EoS definitely overestimates the antiproton yield at midrapidity in this energy range, while the EoS with the crossover transition quite reasonably agrees with the experimental data. Note that the antiprotons are mainly produced from the fireball (baryonless) fluid [39]. To a certain extent, this may be interpreted as being due to multi-meson formation of $p \bar{p}$ in equilibrium in analogy to HSD/PHSD where these channels are not in full equilibrium. The difference between the two EoS's is clearly seen at higher energies $\sqrt{s_{N N}} \geq 20 \mathrm{GeV}$, where the crossover EoS is 
favorable for all hadronic species rather than only for antibaryons $\left(\bar{p}, \bar{\Lambda}, \bar{\Xi}^{+}\right)$.

\subsection{Directed flow in the 3FD model}

In recent works $[44,45,46,47]$ an analysis of the major part of bulk observables has been performed: the baryon stopping [44], yields of different hadrons, their rapidity and transverse momentum distributions [45, 46], as well as the elliptic flow excitation function [47]. This analysis has been carried out for the hadronic EoS and two types of EoS with deconfinement transition: a first-order phase transition and a crossover. It was found that scenarios with deconfinement transitions are preferable especially at high collision energies, though are not perfect.

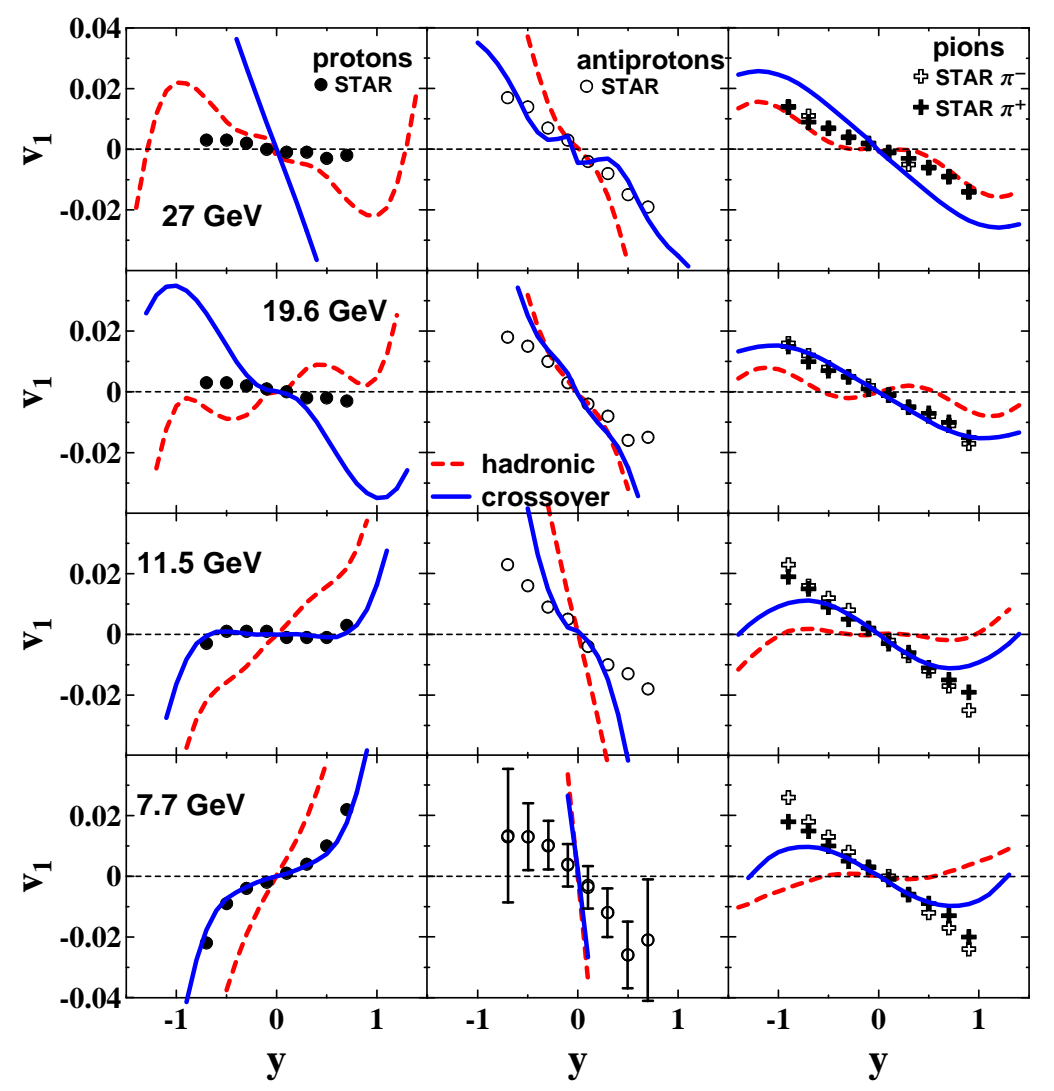

Figure 6: Rapidity distributions of the directed flow for protons, antiproton and positive and negative pions from $\mathrm{Au}+\mathrm{Au}$ collisions at different collision energies calculated within the 3FD model. The experimental data are from the STAR collaboration [21]. The dashed lines correspond to a hadronic EoS while the solid lines stand for a crossover transition.

In this study we consider only two of the above-mentioned scenarios, i.e. the purely hadronic scenario and the crossover one. The reason is primarily technical: It turned out that calculations of the directed flow are demanding and require a high numerical accuracy. In contrast to other observables, the directed flow is very sensitive to the step width of the computational grid and the number of test particles ${ }^{1}$. Therefore, accurate calculations require very high memory and CPU time

\footnotetext{
${ }^{1}$ A numerical "particles-in-cell" scheme is used in the present simulations, see Ref. [39] and references therein for more details. The matter transfer due to pressure gradients, friction between fluids and production of the fireball fluid, is
} 
and accordingly, calculations for a first-order-transition EoS are not completed yet. In particular, due to the same reason we failed so far to perform calculations for energies above $\sqrt{s_{N N}}=30$ $\mathrm{GeV}$. Note that the change of other observables, analyzed so far [44, 45, 46, 47], is below $15 \%$ as compared to results of previous calculations.

The directed flow $v_{1}(y)$ as a function of rapidity $y$ at BES-RHIC bombarding energies is presented in Fig. 6 for pions, protons and antiprotons. As seen, the 3FD model does not perfectly describe the $v_{1}(y)$ distributions. However, we can definitely conclude that the description of the STAR data is better with the crossover EoS than that with the purely hadronic EoS.

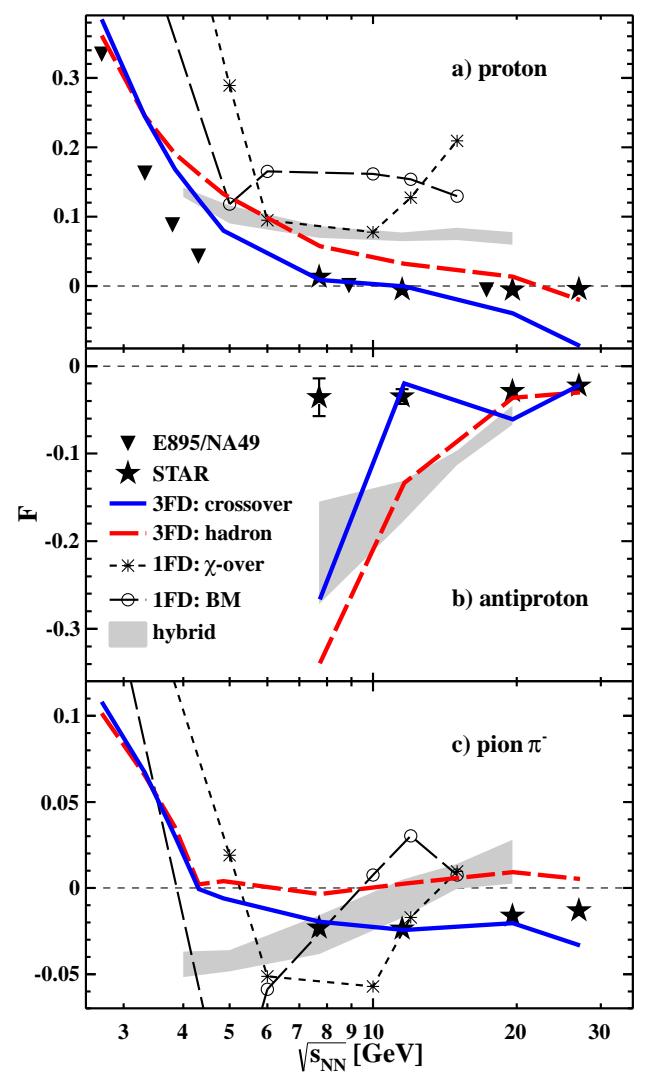

Figure 7: The beam energy dependence of the directed flow slope at midrapidity for protons. The lines are calculated within the 3FD model with a hadronic (dotted lines) and a crossover (solid lines) EoS. For comparison the results of calculations in other collective models are taken from [22]. The experimental data are from the STAR measurements [21] and prior experiments with comparable acceptance cuts [32, 33]. Note the different scales as compared to Fig. 3.

The excitation functions for the slopes of the $v_{1}$ distributions at midrapidity are presented in Fig. 7. As noted above, the discrepancies between experiment and the 3FD model predictions are larger for the purely hadronic EoS (dashed line) and, in addition, some weak substructure is observed here for protons and pions (for example at $\sqrt{s_{N N}}=19.8 \mathrm{GeV}$ ). Indeed, the agreement with the 3FD model for the crossover EoS looks better (solid line in Fig. 7) though it is far from being perfect. Similarly to the kinetic approaches, hydrodynamics has a problem with the description 
of the low energy behavior of the directed flow, however, the boundary of this disagreement shifts down to $8 \mathrm{GeV}$ as compared to $\sqrt{s_{N N}} \sim 20 \mathrm{GeV}$ in the case of PHSD (cf. Fig. 3).

In Ref. [18] an essential part of the STAR data (for $\sqrt{s_{N N}} \leq$ ) $20 \mathrm{GeV}$ is analyzed within collective approaches: the one fluid (1F) hydrodynamical model with a first-order phase transition simulated by the bag model (BM) and a crossover chiral transition ( $\chi$-over), as well as within a modern hybrid model combining hydrodynamics with a kinetic model in the initial and final (afterburner) stages of the collision using both EoS's mentioned above. The results of this work are also displayed in Fig. 7 for comparison (the open circles and stars).

The 3FD model predicts reasonable results for the proton slopes in the range $\sqrt{s_{N N}}<20 \mathrm{GeV}$ for the crossover EoS; the pure hadronic EoS results in a similar energy dependence but with slopes $F_{p}$ exceeds the experimental ones by $\sim 0.2$. A similar behavior is observed for the pion slope function (see Fig. 7). In the case of antiprotons the slope for the crossover EoS (solid line in Fig. 7) is well described at above $10 \mathrm{GeV}$ but it sharply goes down with decreasing energy. For the pure hadronic EoS the 3FD functional dependence of the antiproton slope (dashed line in Fig. 7) looks similarly but is shifted by almost $2-10 \mathrm{GeV}$ towards higher energies.

The results of Ref. [22] for the proton slopes in the 1FD model overestimate the measured ones by an order of magnitude for both chiral ( $\chi$-over) and BM EoS; appropriate results for antiprotons are not reported. The calculational results are more definite for the hybrid model [22]: the shaded region in Fig. 7, that covers predictions for both EoS's, is quite close to the 3FD results with the pure hadronic EoS for protons and antiprotons rather than to the experiment. One can conclude that the fluid dynamical calculations presented in Ref. [18] are not able to explain the observed directed flow of identified hadrons.

\section{Conclusions}

In this study the parton-hadron-string dynamics (PHSD) approach has been applied for the analysis of the recent STAR data on the directed flow of identified hadrons [21] in the energy range $\sqrt{s_{N N}}=7.7-39 \mathrm{GeV}$. The excitation functions for the directed flows of protons, antiprotons and charged pions turn out to be smooth functions in bombarding energy without "wiggle-like" irregularities as expected before in Refs. [34, 35, 36, 37, 38, 48, 49, 50]. Our results differ from the standard UrQMD model at lower bombarding energies as included in Ref. [21] and the recent theoretical analysis in Ref. [22]. The microscopic PHSD transport approach reproduces the general trend in the differential $v_{1}(y)$ excitation function and leads to an almost quantitative agreement for protons, antiprotons and pions especially at higher energies. We attribute this success to the Kadanoff-Baym dynamics incorporated in PHSD (with more accurate spectral functions) as compared to a Boltzmann-like on-shell transport model (UrQMD) and the account for parton dynamics also in this "moderate" energy range. The latter is implemented in PHSD in line with an equation of state from lattice QCD [51]. The formation of the parton-hadron mixed phase softens the effective EoS in PHSD and describes a crossover transition (in line with the lattice QCD EoS). Accordingly, the PHSD results differ from those of HSD where no partonic degrees of freedom are incorporated. A comparison of both microscopic models has provided detailed information on the effect of parton dynamics on the directed flow (cf. Fig. 2). 
Antiprotons have been shown to be particularly interesting. In HSD/PHSD we include antiproton annihilation into several mesons while taking into account also the inverse processes of $p \bar{p}$ creation in multi-meson interactions by detailed balance [30]. Related kinetic models (including UrQMD) which neglect the inverse processes for antiproton annihilation at lower energies do not describe the data on the directed flow of hadrons $v_{1}(y)$. Note that 3FD hydrodynamics provides the best results with a crossover EoS for the quark-hadron phase transition which by default is implemented in PHSD.

Still sizeable discrepancies with experimental measurements in the directed flow characteristics are found for the microscopic kinetic models at $\sqrt{s_{N N}} \leqslant 20 \mathrm{GeV}$ and are common for both HSD and PHSD (and UrQMD [52]) since the partonic degrees of freedom are subleading at these energies. We recall that the flow observables are not only ones where the kinetic approaches have a problem in this energy range. Another long-standing issue is the overestimation of pion production as seen in Fig. 1 in the energy regime around the 'horn' in the $K^{+} / \pi^{+}$meson ratio $[53,54]$ which before has been related to a first order phase transition or to the onset of deconfinement [55]. Our flow analysis shows no indication of a first order transition such that the question addressed in the title of this work has to be answered with: no! However, we have found further strong evidence that the dynamics of heavy-ion reactions at lower SPS and AGS energies is far from being understood especially on the hadronic level. We speculate that extended approaches including consistently chiral partners as well as a restoration of chiral symmetry at high baryon density and/or temperature might lead to a solution of the problem as well as precise experimental studies at FAIR and NICA [5].

\section{Acknowledgments}

This work in part was supported by the LOEWE center HIC for FAIR as well as by BMBF. V.D.T. was also partially supported by the Heisenberg-Landau grant of JINR. Y.B.I. was partially supported by grant NS-932.2014.2.

\section{References}

[1] S. A. Voloshin, A. M. Poskanzer and R. Snellings, in Landolt-Boernstein New Series, I/23, p. 5-54, edited by R. Stock (Springer-Verlag, 2010).

[2] P. Sorensen, In Quark-Gluon Plasma 4, ed. by R. Hwa and X.N. Wang, World Scientific (2010).

[3] A. M. Poskanzer and S. A. Voloshin, Phys. Rev. C 58, 1671 (1998).

[4] M.M.Aggarwal et al. (STAR Collaboration), arXiv:1007.2613.

[5] P. Senger et al., Lect. Notes Phys. 814, 681 (2011).

[6] H. Sorge, Phys. Rev. Lett. 78, 2309 (1997).

[7] N. Herrmann, J. P. Wessels, and T. Wienold, Ann. Rev. Nucl. Part. Sci. 49, 581 (1999).

[8] E. Schnedermann and U. Heinz, Phys. Rev. Lett. 69, 2908 (1992).

[9] D. E. Kahana et al., Phys. Rev. Lett. 74, 4404 (1995).

[10] J. Barrette et al., (E877 Collaboration), Phys. Rev. Lett. 73, 2532 (1994). 
[11] I. Bearden et al., (NA44 Collaboration), Phys. Rev. Lett. 78, 2080 (1997).

[12] V. P. Konchakovski et al., Phys. Rev. C90, 014903 (2014).

[13] J. Adams et al. (STAR Collaboration), Phys. Rev. Lett. 92, 062301 (2004); A.H. Tang (STAR Collaboration), J. Phys. G 30, S1235 (2004); J. Adams et al., Phys. Rev. C 72, 014904 (2005); J. Adams et al., Phys. Rev. C 73, 034903 (2006). B. I. Abelev et al., Phys. Rev. Lett. 101, 252301 (2008); Y. Pandit (STAR Collaboration), J. Phys. Conf. Ser. 316, 012001 (2011).

[14] M. Belt Tonjes (PHOBOS Collaboration), J. Phys. G30, S1243 (2004); B. B. Back et al. (PHOBOS Collaboration), Phys. Rev. Lett. 97, 012301 (2006).

[15] P. Bozek and I. Wyskiel, Phys. Rev. C 81, 054902 (2010).

[16] V. D. Toneev et al., Phys. Rev. C 85, 034910 (2012).

[17] A. V. Merdeev, L. M. Satarov, and I. N. Mishustin, Phys. Rev. C 84, 014907 (2011).

[18] J. Steinheimer et al.,Phys. Rev. C 81, 044913 (2010).

[19] J. Y. Chen et al., Phys. Rev. C 81, 014904 (2010).

[20] H. Petersen, Q. Li, X. Zhu, and M. Bleicher, Phys. Rev. C 74, 064908 (2006).

[21] L. Adamczyk, et al. (STAR Collaboration), Phys. Rev. Lett. 112, 162301 (2014).

[22] J. Steinheimer et al., Phys. Rev. C 89, 054913

[23] S. Juchem, W. Cassing, and C. Greiner, Phys. Rev. D 69, 025006 (2004); Nucl. Phys. A 743, 92 (2004).

[24] W. Cassing and E. L. Bratkovskaya, Nucl. Phys. A 831, 215 (2009); Phys. Rev. C 78, 034919 (2008); W. Cassing, Nucl. Phys. A 791, 365 (2007).

[25] W. Cassing, E. Phys. J. ST 168, 3 (2009).

[26] V. P. Konchakovski et al., Phys. Rev. C 85, 011902 (2012).

[27] A. Andronic, P. Braun-Munzinger and J. Stachel, Nucl. Phys. A 772, 167 (2006).

[28] X. Zhu (STAR Collaboration), Acta Phys. Polon. Supp. 5, 213 (2012).

[29] C. Blume and C. Markert, Prog. Part. Nucl. Phys. 66, 834 (2011).

[30] W. Cassing, Nucl. Phys. A 700, 618 (2002).

[31] S. A. Bass et al., Prog. Part. Nucl. Phys. 42, 279 (1998).

[32] C. Alt et al. (NA49 Collaboration), Phys. Rev. C 68, 034903 (2003).

[33] H. Liu et al. (E895 Collaboration), Phys. Rev. Lett. 84, 5488 (2000).

[34] L. P. Csernai and D. Rohrich, Phys. Lett. B 458, 454 (1999).

[35] J. Brachmann et al., Phys. Rev. C 61, 024909 (2000).

[36] D. H. Rischke et al., Heavy Ion Phys. 1, 309 (1995).

[37] D. H. Rischke, Nucl. Phys. A 610, 88 (1996).

[38] H. Stöcker, Nucl. Phys. A 750, 121 (2005).

[39] Yu. B. Ivanov, V. N. Russkikh, and V. D. Toneev, Phys. Rev. C 73, 044904 (2006).

[40] Y. B. Ivanov, Yad. Fiz. 46, 100 (1987) [Sov. J. Nucl. Phys. 46, 63 (1987)]. 
[41] Yu.B. Ivanov, Nucl. Phys. A 474, 669 (1987).

[42] V. M. Galitsky and I. N. Mishustin, Sov. J. Nucl. Phys. 29, 181 (1979).

[43] A. S. Khvorostukhin et al., Eur. Phys. J. C 48, 531 (2006).

[44] Yu. B. Ivanov, Phys. Rev. C 87, 064904 (2013).

[45] Yu. B. Ivanov, Phys. Rev. C 87, 064905 (2013).

[46] Yu. B. Ivanov, Phys. Rev. C 89, 024903 (2014).

[47] Yu. B. Ivanov, arXiv:1401.2265; Yu. B. Ivanov, Phys. Lett. B 723, 475 (2013).

[48] C. M. Hung and E. V. Shuryak, Phys. Rev. Lett. 75, 4003 (1995).

[49] A. A. Shanenko and V. D. Toneev, JINR Rap. Com.5[73], 21 (1995); E.G. Nikonov, A.A. Shanenko and V.D. Toneev, Heavy Ion Phys. 4, 333 (1996).

[50] D. Rischke and M. Guylassy. Nucl. Phys. A A97, 4 (1996).

[51] Y. Aoki et al., Phys. Lett. B 643, 46 (2006); S. Borsanyi et al., JHEP 1009, 073 (2010); JHEP 1011, 077 (2010); JHEP 1208, 126 (2012); Phys. Lett. B 730, 99 (2014).

[52] E. L. Bratkovskaya et al., Phys. Rev. C 69, 054907 (2004).

[53] W. Cassing, E. L. Bratkovskaya and S. Juchem, Nucl. Phys. A 674, 249 (2000).

[54] M. Gazdzicki and M. I. Gorenstein, Acta Phys. Polon. B 30, 2705 (1999).

[55] M. Gazdzicki, M. Gorenstein and P. Seyboth, Acta Phys. Polon. B 42, 307 (2011). 